\title{
The world's first human-to-human heart transplant at Groote Schuur Hospital: 50 years later
}

Fifty years ago, on 3 December 1967, the world's first human-tohuman heart transplant was performed by Dr Christiaan Barnard at Groote Schuur Hospital in Cape Town. This was, and probably will remain, the most publicised medical event of all time, making headlines in nearly every international newspaper, magazine and tabloid within days of the event. The idea of transplanting a heart from one human to another captured the minds and imaginations of the public like no other medical event before or since. The only other iconic event in that era that was equally well publicised was man's first landing on the moon 18 months later.

This medical breakthrough was published in the SAMJ 3 weeks later, towards the end of December 1967, and is one of the most cited articles in this journal.

The first heart transplant placed Groote Schuur Hospital, the University of Cape Town and South Africa (SA) firmly on the international medical map. A heart transplant was seen by the public as transplanting the very soul of an individual from one person into another. It provoked tremendous debate, some of it very emotional, around the ethics of transplantation and spurred on the international medical and philosophy community to develop the concept of brain death into law. Many countries took decades to adopt such laws - most notably Japan, which took another 30 years (until 1997) to allow organ transplantation from brain-dead donors. Even today many countries do not accept brain death, preventing organ transplantation as a therapeutic option for end-stage heart failure.

This tremendous publicity generated by the first heart transplant occurred despite kidney and liver transplantation having preceded heart transplantation by many years. These prior surgical innovations were instrumental in paving the way for immunosuppression, vital for modifying the recipient immune response and preventing rejection from a genetically non-identical donor. The suppression of host rejection as well as the prevention and treatment of subsequent side-effects remains the biggest challenge in organ transplantation.

Ultimately, the goal of a transplant is to improve both length and quality of life when medication and conventional cardiac surgery will not adequately alleviate symptoms of heart failure. As far as possible, transplant teams strive to help the recipient lead a life similar in quality to that of his or her peers, unconstrained by the limitations of heart failure.

The operation itself as performed today has changed very little since Dr Norman Shumway of Stanford University in the USA first described the technique in animals in the early 1960s. Small technical variations, the use of heart-preservation solutions (cardioplegia), and improvements in the heart-lung machine have optimised this procedure through the decades. The lack of donors has also been offset by the use of implantable mechanical assist devices (miniature pumps that help the heart), and even a totally artificial heart, as a bridge to transplantation, as well as the recent use of donors whose hearts have stopped (donation after circulatory death: DCD), which would previously have been deemed unsuitable for transplantation. The expensive equipment required to resuscitate these DCD hearts is not available in SA at present, however, and mechanical assist devices are currently only accessible to patients with excellent health insurance. In those developed countries that can afford their extended use, mechanical assist devices are increasingly being used as

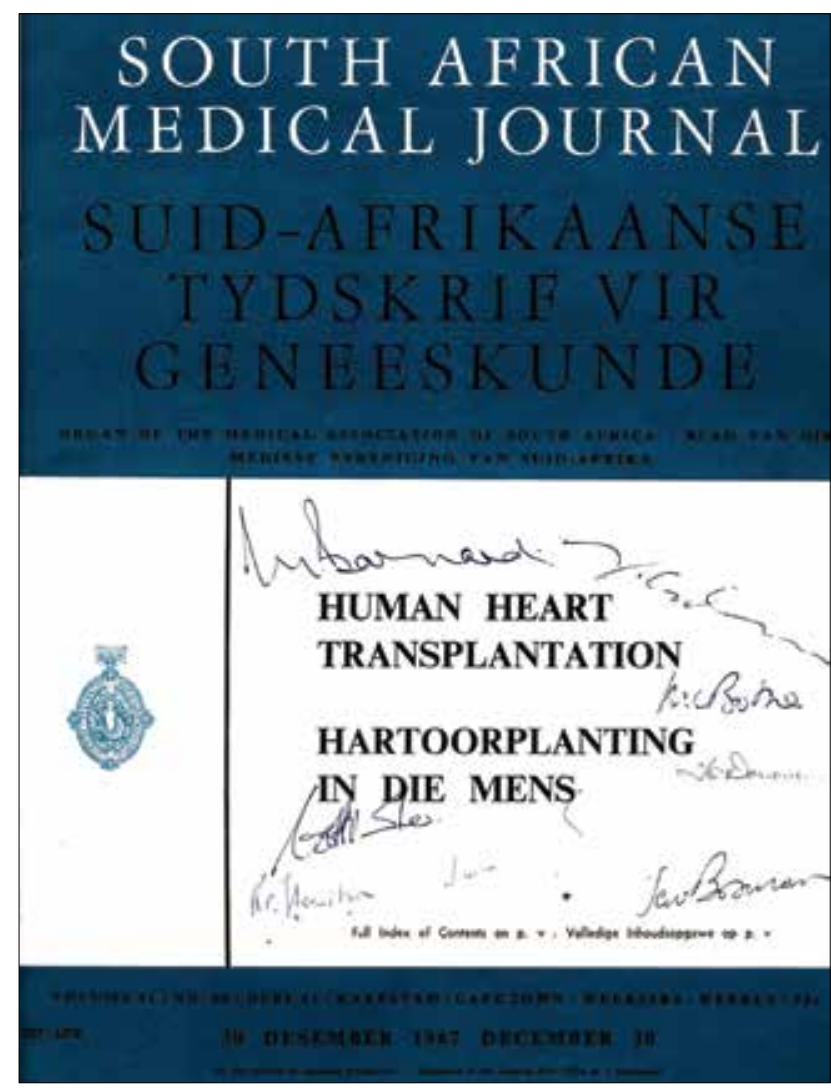

The cover of the SAMJ of 30 December 1976, signed by some of the members of the heart transplant team. From the top in a clockwise direction, the signatures are those of Prof. Chris Barnard, Prof. Val Schrire (cardiologist and head of the cardiac clinic at Groote Schuur Hospital/University of Cape Town), Dr M C Botha (haematologist/immunologist), Dr Terry O'Donovan (surgeon), Dr S C Bosman (surgeon), Dr Joe (Oz) Ozinsky (anaesthetist), Dr Rodney Hewitson (senior surgeon), and Dr Coert Venter (junior surgeon in training). Two key articles from the issue are reprinted in this edition of SAMJ.

permanent treatment for heart failure, so-called destination therapy, because of the dearth of donor organs.

Progress in postoperative critical care has significantly improved early outcomes of heart transplantation, and patients without complications are discharged from the intensive care unit in 4 days or less. In the past year Groote Schuur Hospital has instituted a longterm extracorporeal membrane oxygenation (ECMO) service, which brings the unit in line with international standards for postoperative care in complicated cases.

Approximately 15 years after the first heart transplant, in the early 1980s a 'miracle' drug called cyclosporine was discovered by Jean Borel, earning him a Nobel Prize. This drug was a significant breakthrough in the management of donor organ rejection, and transformed transplantation from an experimental operation in leading academic medical centres to standard medical treatment for end-stage organ failure with reproducible results that could be applied more widely. The numbers of all organ transplants rapidly escalated in the early 1980s, reaching a plateau a decade later when the availability of donor organs became the major constraint. Cyclosporine is a member of the class of drugs called calcineurin 
inhibitors (Sandimmun and Neoral are the commercial formulations of the drug), and the other commonly used drug in this class is tacrolimus (Prograft or Advagraft).

Calcineurin inhibitors are still the mainstay of treatment in organ transplantation, and their use has led to significantly fewer rejection episodes and longer life expectancy. However, unwanted sideeffects are a concern with this more effective immunosuppression. Increased incidences of infection, hypertension, nephrotoxicity and in some instances long-term renal failure, diabetes, high cholesterol and cancers are some of the side-effects of immunosuppression. The newer immunosuppressive drugs show promise of a reduction in some of these side-effects with or without some calcineurin inhibition, and provide physicians with a larger armamentarium to treat patients. The medical management of heart transplant recipients remains an ongoing challenge during the period immediately after the transplant, as well as in the long term.

Following the significant milestone 50 years ago, the Christiaan Barnard Division of Cardiothoracic Surgery at the University of Cape Town and Groote Schuur and Red Cross War Memorial Children's hospitals has transplanted 537 hearts and remains the only heart transplant facility for both indigent and insured patients in SA. Despite the challenges that exist with heart transplantation, the outcome has improved significantly over the past 20 years. The functional status of the recipient after the procedure is generally excellent, depending on his or her motivation, with an expected $85 \%$ survival rate at 1 year, decreasing to $75 \%$ at 5 years.

The Christiaan Barnard Division of Cardiothoracic Surgery is the proud host of an international celebration to commemorate the 50th anniversary of the world's first heart transplant, ' 50 years of Heart Transplantation: Courage and Innovation', on 2 - 4 December 2017 at Groote Schuur Hospital.

We hope that the celebration of this courageous and innovative event will not only encourage further innovations in the management of heart failure, which constitutes a major burden of disease, but also inspire young academics to follow in the footsteps of Christiaan Barnard and his team five decades later.
Johan Brink

Christiaan Barnard Division of

Cardiothoracic Surgery, Groote Schuur Hospital and Faculty of Health Sciences, University of Cape Town, South Africa johan.brink@uct.ac.za

\section{Tim Pennel}

Christiaan Barnard Division of

Cardiothoracic Surgery, Groote Schuur Hospital and Faculty of Health Sciences, University of Cape Town, South Africa

\section{Karen Seele}

Christiaan Barnard Division of

Cardiothoracic Surgery, Groote Schuur Hospital and Faculty of Health Sciences, University of Cape Town, South Africa

\section{Peter Zilla}

Christiaan Barnard Division of

Cardiothoracic Surgery, Groote Schuur Hospital and Faculty of Health Sciences, University of Cape Town, South Africa
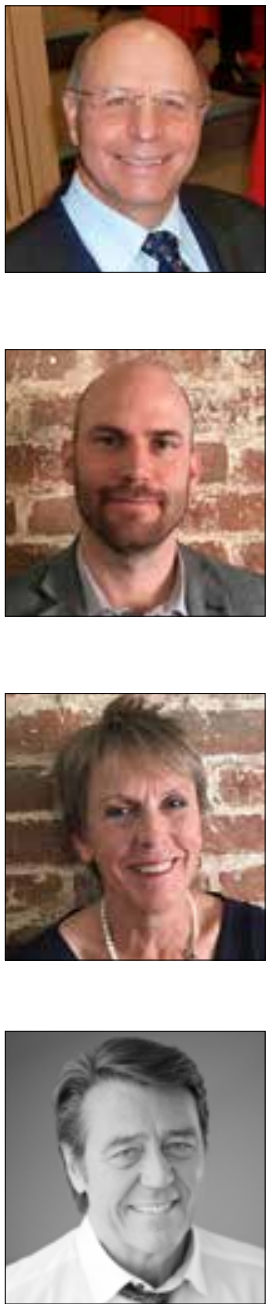

S Afr Med J 2017;107(12):1035-1036. DOI:10.7196/SAMJ.2017.v107i12.12960

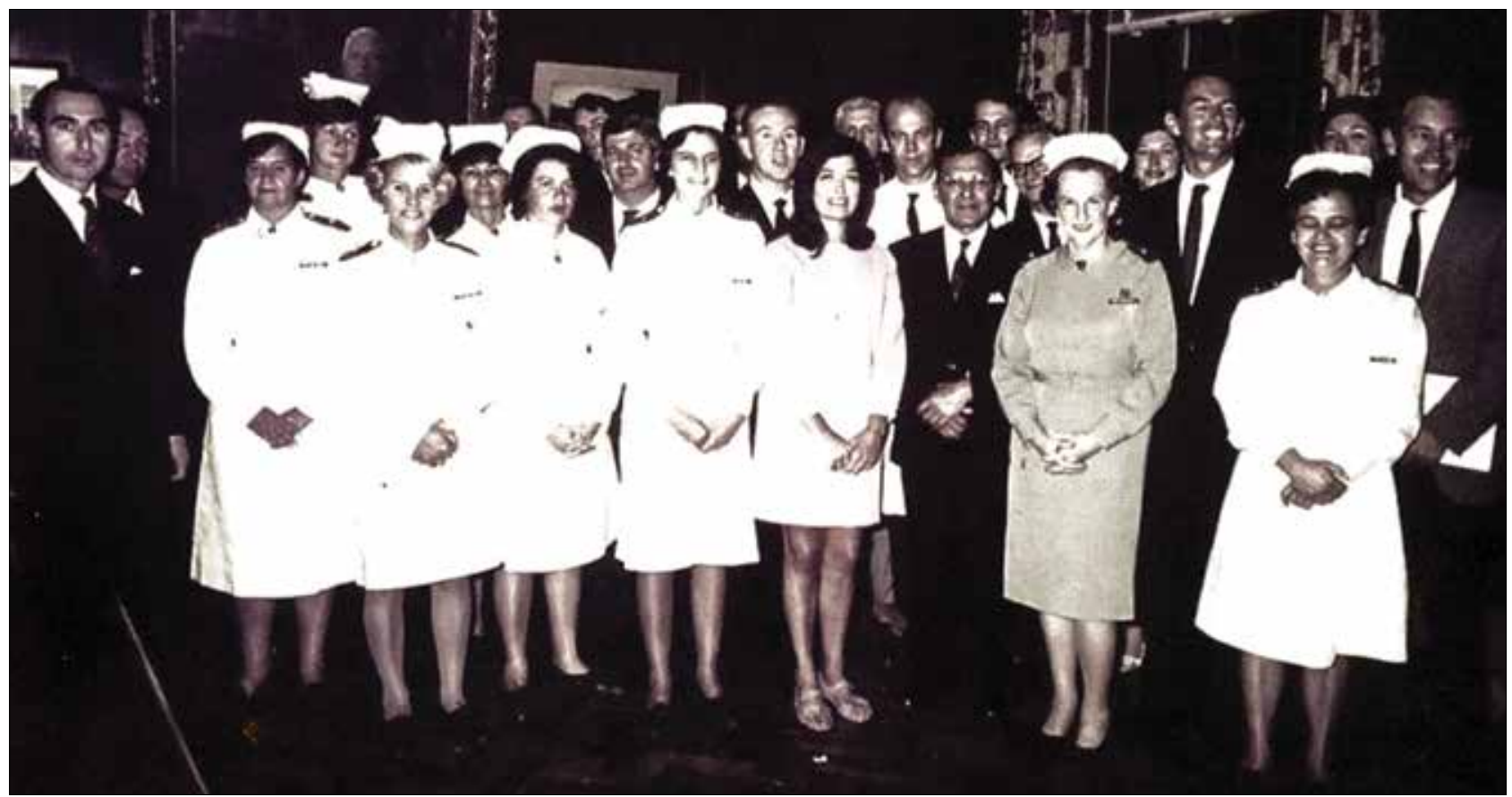

Some of the members of the team that performed the first heart transplant, photographed on 3 December 1967 (photo: First Heart Transplant Museum, Groote Schuur Hospital). The full team is listed on p. 1037 of this issue. 